\title{
Randomized controlled trials in surgery: are we still missing something important?
}

\author{
Michael Moen
}

Received: 4 April 2012 / Accepted: 4 April 2012 /Published online: 10 May 2012

(C) The International Urogynecological Association 2012

The goal of clinical research is to answer questions about treatment in order to improve patient care. Clinical research falls into two basic categories: observational and experimental. Evidence-based medicine (EBM) compels clinicians to utilize best available information to guide clinical decisionmaking and is recognized as the most important method to improve the effectiveness and safety of patient care. Although information can be derived from both observational and experimental studies, the randomized controlled trial (RCT) provides the highest quality of evidence in clinical research and is the cornerstone of EBM.

The vast majority of studies in surgery, however, are observational, not experimental, and the need for more RCTs is well-recognized. Unfortunately, performing highquality RCTs in surgery remains a difficult task with many obstacles which are not typically encountered in nonsurgical trials. In addition, several factors have been identified which indicate there is a need to improve the overall quality of surgical RCTs [1-3]. Many of the potential weaknesses of surgical RCTs are related to the nature of surgical practice itself, which inherently introduces more variables and potential biases. The overall quality of RCTs varies greatly based on the ability to account for these potential biases in the design and conduct of individual trials.

Thankfully, the number of RCTs in urogynecologic surgery has steadily increased. However, as research in urogynecology has progressed, several potential weaknesses have been identified which can affect the quality of RCTs in urogynecology. Strategies to account for these potential weaknesses have

\section{Moen $(\triangle)$}

Illinois Urogynecology, Ltd,

1875 Dempster St, Suite 665,

Park Ridge, IL 60068, USA

e-mail: Michael.Moen-MD@Advocatehealth.com evolved over the past several years, resulting in improved quality of urogynecologic RCTs. Such strategies include confirmation that randomization resulted in similar comparison groups in terms of patient characteristics, demographics, and concurrent procedures, performing blinded assessments of anatomic outcomes, providing patient flow diagrams, and accounting for missing data and loss to follow-up with bestcase and worst-case analyses.

Another important strategy has been the use of multiple outcome measures to define success and failure in pelvic organ prolapse (POP) surgery. Many early RCTs used strictly anatomic measures to define success, which resulted in reports of high failure rates for surgical procedures. Recognizing the importance of these changes in reporting outcomes has even prompted reevaluation and rereporting of important previous studies concerning POP surgery $[4,5]$.

One of the greatest difficulties in surgical RCTs is achieving adequate sample size to allow meaningful statistical analysis. It can be difficult, if not impossible, to recruit sufficient numbers of appropriate subjects within a reasonable time frame to conduct a valid study. The need to achieve adequate sample size has resulted in one of the most important strategies to improve surgical research, the evolution of the multisite RCT. Many authors refer to the multisite RCT design as a "strength" when describing the study, typically including the comment that "generalizability" is improved with this design. However, from a research methodology standpoint, adding multiple surgeons is a confounder that adds bias due to the potential for variation in surgical technique between surgeons and is thus a potential weakness which must be accounted for in the study. Surgery requires a requisite level of performance that is dependent on the acquisition of skill and experience which, in itself, is highly variable. It varies from surgeon to surgeon, it varies for individual surgeons for different procedures, it varies for 
individual surgeons over time, and it varies case by case based on individual patient factors. The bottom line is that the surgeon is an important variable in surgery and this critical issue must be recognized and accounted for in surgical research.

Surgical technique is often addressed in the design and methods of surgical RCTs, but it is rarely analyzed and reported once data have been collected. This is a major flaw in current urogynecologic research. Individual surgeon performance should be analyzed to assure subjects truly received similar intervention throughout the trial. Differences in surgeon skill and experience can introduce a form of performance bias which could result in subjects receiving a greater variation in surgical intervention than intended by the study design. Without analyzing for potential performance bias, the RCT is severely flawed and the results provided may be inaccurate and misleading. This is a critical issue because of the importance placed on RCTs in guiding changes in clinical practice.

Although rarely addressed in individual surgical trials, examples of performance bias exist within the current urogynecologic literature and the potential for performance bias is not limited to multisite RCTs. In fact, the issue of surgeon factors was raised after the landmark first RCT in POP surgery by Benson et al. [6]. Many subsequent authors have referenced this study as Level I evidence that abdominal surgery is superior to vaginal surgery for POP. However, the subsequent criticism was that the results of the study simply indicated Dr. Benson was better at performing sacrocolpopexy than sacrospinous ligament fixation, not that abdominal surgery was better than vaginal surgery [7].

Another important example of performance bias in the urogynecology literature is the landmark study by Ward et al. comparing tension-free vaginal tape (TVT) to colposuspension, which involved 14 surgeons [8]. The conclusions from this trial contributed to a significant change in practice patterns concerning surgical management of stress incontinence. In a subsequent report, Hilton [9] provided additional data which confirmed surgeon factors had a significant impact on the results of the trial. The range of success by site in the trial ranged from 0 to $90 \%$ for TVT and 0 to $92 \%$ for colposuspension. Furthermore, the success rate for both procedures was highest in the centers which recruited the most patients, indicating surgeon experience likely explained the differences in outcomes. It was acknowledged this indicated a level of performance below the expected threshold for some centers, confirming the importance of accounting for surgeon factors in the analysis of the study.

A decade later, we are in the midst of continued debate in determining the "best" surgical treatment for POP. Several RCTs in POP surgery involve various comparisons of the three main approaches to POP surgery: native tissue repairs, abdominally placed mesh, and vaginally placed mesh. A concern is that performance bias is a potential issue in determining the true success rates of non-mesh repairs and in determining the rate of morbidity related to meshaugmented repairs. Two important studies which recently reported relatively high morbidity with mesh have been questioned. In one, surgeons were noted to have a different level of experience in the two procedures being compared (three times the number of cases for one procedure than the other) and subsequently had a higher than expected rate of morbidity in the procedure in which there was less experience [10]. In another study, which involved 22 surgeons, the authors reported a wide range of outcomes by surgeons including 0 $100 \%$ failure rates for both the experimental and control groups and also $0-100 \%$ for the complication rate of mesh exposure [11]. A subsequent report by the same authors which included subjects from the first trial proved surgeon experience had a direct impact on improved outcomes [12].

In summary, many factors have resulted in improved quality of RCTs in urogynecologic surgery; however, the potential impact of surgeon factors has remained missing from the vast majority of studies. Without accounting for surgeon factors, a trial could be severely flawed and might not provide an accurate comparison between procedures. Ideally, the range of outcomes for individual surgeons should be measured, analyzed, and reported for all surgical RCTs, providing a more formal analysis of generalizability with best-case and worst-case scenarios based on surgeon performance. At minimum, it should be confirmed once data have been collected that all surgeons participating in the study had passed through their own learning curve to reach a level of anticipated acceptable performance, assuring subjects truly received similar intervention throughout the trial. It is time to recognize the importance of individual surgeon performance in surgical research and routinely include formal evaluation of surgeon factors in surgical RCTs.

\section{References}

1. McCulloch P, Taylor I, Sasako M, Lovett B, Griffin D (2002) Randomised trials in surgery: problems and possible solutions. BMJ 324:1448-1451

2. Dimick JB, Diener-West M, Lipsett PA (2001) Negative results of randomized clinical trials published in the surgical literature: equivalency or error? Arch Surg 136:796-800

3. Chang DC, Matsen SL, Simpkins CE (2006) Why should surgeons care about clinical research methodology? J Am Coll Surg 203:827-830

4. Weber AM, Walters MD, Piedmonte MR, Ballard LA (2001) Anterior colporrhaphy: a randomized trial of three surgical techniques. Am J Obstet Gynecol 185:1299-1306

5. Chmielewski L, Walters MD, Weber AM, Barber MD (2011) Reanalysis of a randomized trial of 3 techniques of anterior colporrhaphy using clinically relevant definitions of success. Am J Obstet Gynecol 205:69.e1-69.e8

6. Benson JT, Lucente V, McClellan E (1996) Vaginal versus abdominal reconstructive surgery for the treatment of pelvic support 
defects: a prospective randomized study with long-term outcome evaluation. Am J Obstet Gynecol 175:1418-1422

7. Anonymous (1999) Response to letter to the editor. J Pelvic Surg 5:309-313

8. Ward K, Hilton P, United Kingdom and Ireland Tension-free Vaginal Tape Trial Group (2002) Prospective multicentre randomised trial of tension-free vaginal tape and colposuspension as primary treatment for stress incontinence. BMJ 325:67

9. Hilton P (2002) Trials of surgery for stress incontinence - thoughts on the 'Humpty Dumpty principle'. BJOG 109:1081-1088
10. Iglesia CB, Sokol AI, Sokol ER, Kudish BI, Gutman RE, Peterson JL, Shott S (2010) Vaginal mesh for prolapse: a randomized controlled trial. Obstet Gynecol 116:293-303

11. Withagen MI, Milani AL, den Boon J, Vervest HA, Vierhout ME (2011) Trocar-guided mesh compared with conventional vaginal repair in recurrent prolapse: a randomized controlled trial. Obstet Gynecol 117:242-250

12. Withagen MI, Vierhout ME, Hendriks JC, Kluivers KB, Milani AL (2011) Risk factors for exposure, pain, and dyspareunia after tension-free vaginal mesh procedure. Obstet Gynecol 118:629-636 